\title{
Optimizing Antibiotic Pharmacodynamics for Clinical Practice
}

\author{
Kevin P Connors, Joseph L Kuti* and David P Nicolau
}

Center for Anti-Infective Research and Development, Hartford Hospital, Hartford Connecticut, USA

\begin{abstract}
With an absence of new antibiotics in the pipeline to address increasing resistance among gram-positive and gram-negative bacteria, new strategies are needed to retain utilization of currently available agents. As recommended in current guidelines for antimicrobial stewardship, dosage optimization through consideration of antibiotic pharmacodynamics provides a formidable approach to making good antibiotics better. Knowledge of the relationship between antibiotic concentration to microbiological potency and its effect on antibacterial activity leads to the design of dosing regimens that optimize killing of bacteria in the clinical setting. The activity of aminoglycosides is dependent on maximizing peak free drug concentrations in relation to their minimum inhibitory concentration (MIC), so giving larger doses less frequently has become the gold-standard strategy for optimizing their pharmacodynamics. In contrast, the activity of $\beta$-lactam antibiotics is dependent on maximizing the time that free drug concentrations remain above the MIC; numerous approaches including continuous and prolonged infusion of these agents enable optimization of this pharmacodynamics parameter and improve clinical outcomes. This review discussed pharmacodynamics concepts as applied clinically for aminoglycosides, $\beta$-lactams, and other classes of antibiotics.
\end{abstract}

Keywords: Antibiotics; Pharmacodynamics; Optimization; Aminoglycoside; Beta-Lactams; Prolonged Infusion; Continuous infusion; Stewardship

\section{Introduction}

In recent decades, emerging bacterial resistance is defying the efficacy of currently available antibiotics. Of the approximate 1.7 million hospital acquired infections in the US annually, a staggering 350,000 infections can be attributed to just a few multi-drug resistant pathogens [1-3]. Current consensus indicates Enterococcus faecium, Staphylococcus aureus, Klebsiella pneumoniae, Acinetobacter baumanii, Pseudomonas aeruginosa, and Enterobacter species, affectionately dubbed the "ESKAPE" pathogens, to be overwhelmingly responsible for the majority of antibiotic resistant infections found in US hospitals [4,5]. In the US alone, methicillin resistant S. aureus (MRSA) infections have been linked to higher mortality rates than HIV/AIDS and tuberculosis combined [6,7]. In addition to increased morbidity and mortality, figures from the Centers for Disease Control and Prevention (CDC) associate drug-resistant infections with an economic burden of approximately US $\$ 3.5$ billion per year [2,3].

Given current trends in infectious disease, it's unfortunate that the scientific, regulatory, and economic challenges of antibiotic research have led to a decline in the approval of new agents [4,5,8-13]. Today, there are few new antibiotics in late stage development offering activity against the most dangerous hospital acquired gram-negative pathogens [14-16]. Of 90 antibacterial agents in development with in vitro activity reviewed by The European Centre for Disease Prevention and Control/European Medicines Agency, only 66 were considered "new agents" of which only 27 showed potential benefits over current agents. Furthermore, just 15 of the 27 agents could be systemically administered, with only 2 agents exhibiting activity against gramnegative pathogens via a new mechanism [14]. Presently there are 6 antibiotics in Phase II-III trials, 3 of which demonstrate activity against Pseudomonas aeruginosa: MK-7655/imipenem-cilastatin (Merck), ceftazidime/avibactam (Astra Zeneca/Forest), and ceftolozane/ tazobactam (Cubist) [15,16].

By limiting inappropriate antimicrobial use and enhancing the selection, dose, route, and duration of therapy, institutions with ongoing antimicrobial stewardship programs are bridging the gap between resistance and optimizing available therapies for favorable clinical outcomes [17-19]. Techniques employed by stewardship teams may include formulary restriction, implementation of specialized order sets, treatment algorithms and guidelines designed specifically for the institution, education of practitioners, pharmacy dosing programs, and pharmacodynamic dose optimization [18]. The last strategy, of which this review will focus, describes the use of pharmacodynamic principles to guide the selection and optimization of the dosing regimen. This review will describe strategies to optimize dosing of some currently available antibiotic classes through the incorporation of pharmacodynamic principles in clinical practice.

\section{Basic Principles of Pharmacodynamics}

A true understanding of the pharmacodynamics begins with a few basic principles. First, administering a fixed dose of drug to a large number of patients will result in substantially different profiles of changing concentrations of the drug over time (i.e., variability in pharmacokinetics). Second, the shape of the curve describing the concentration-time profile can have a direct impact on the effect of a particular drug dose (i.e., different drugs have different pharmacodynamic properties). Only free (non-protein bound) drug is microbiologically active. The higher the value of the measure of the potency of the drug [e.g., the minimum inhibitory concentration (MIC)] for the pathogen, the less effect a fixed drug exposure will have, and finally, it is the drug exposure at the site of the infection which is responsible for the antimicrobial effect. With respect to the latter,

*Corresponding author: Joseph L Kuti, Center for Anti-Infective Research and Development, Hartford Hospital, 80 Seymour Street, Hartford, CT 06102, USA, Tel: (860) 545-3612; Fax: (860)545-3992; E-mail: jkuti@harthosp.org

Received January 17, 2013; Accepted March 26, 2013; Published March 29 2013

Citation: Connors KP, Kuti JL, Nicolau DP (2013) Optimizing Antibiotic Pharmacodynamics for Clinical Practice. Pharmaceut Anal Acta 4: 214 doi:10.4172/2153-2435.1000214

Copyright: (c) 2013 Connors KP, et al. This is an open-access article distributed under the terms of the Creative Commons Attribution License, which permits unrestricted use, distribution, and reproduction in any medium, provided the original author and source are credited. 
most pharmacodynamic studies reference drug exposure in blood to an effect that takes place at the site of infection (e.g., lung epithelial lining fluid, tissue, bone, etc). For most antibiotics, including $\beta$-lactams, the exposure at the site of infection is similar to that of blood, and therefore, blood is a reasonable surrogate marker for exposure. However, one must consider each antibiotics pharmacokinetic characteristic to determine if this relationship will hold true. For example, macrolide antibiotics such as azithromycin have low blood concentrations, a large volume of distribution, and penetrate widely into lung alveolar macrophages; therefore, the exposure in blood is only a correlate for the required exposure needed to achieve an antimicrobial effect in the lung.

One of the first studies elucidating the relationship between drug concentration (i.e., pharmacokinetics) and microbiological effect (i.e., pharmacodynamics) can be traced back to the 1940s when Eagle and colleagues observed the antibacterial properties of penicillin to be related to time and those of streptomycin to be concentration [20]. This concept of time and concentration in relation to drug exposure are paramount to distinguishing the bactericidal activity of antibiotics. Furthermore, this concentration/time exposure profile can be further quantified by characterizing a mathematical relationship with antibacterial effect and the maximum free drug concentration $\left(f \mathrm{C}_{\max }\right) / \mathrm{MIC}$ ratio, the area under the free drug concentration-time curve (fAUC)/MIC ratio, or the time in which free drug concentrations exceed the MIC $(f \mathrm{~T}>\mathrm{MIC})$ with microbiological outcome (Figure 1) [21-23].

At clinically relevant concentrations, antibiotics like $\beta$-lactams (penicillins, cephalosporins, carbapenems, and monobactams), lincosamides, macrolides, oxazolidinones, vancomycin and tigecycline exhibit time-dependent microbiological effects, whereby increases in concentration alone relative to the MIC do not add to enhanced killing of bacteria. For some agents, such as the $\beta$-lactams, optimized killing is obtained when a specific $f \mathrm{~T}>\mathrm{MIC}$ exposure is achieved against the pathogen. This exposure varies by type of $\beta$-lactam, as well as by bacteria. In general, members of the penicillin class require approximately $50 \% f \mathrm{~T}>\mathrm{MIC}$ for maximal exposure, the cephalosporins require $50-70 \% f \mathrm{~T}>\mathrm{MIC}$, and carbapenems require $30-40 \% f \mathrm{~T}>\mathrm{MIC}$

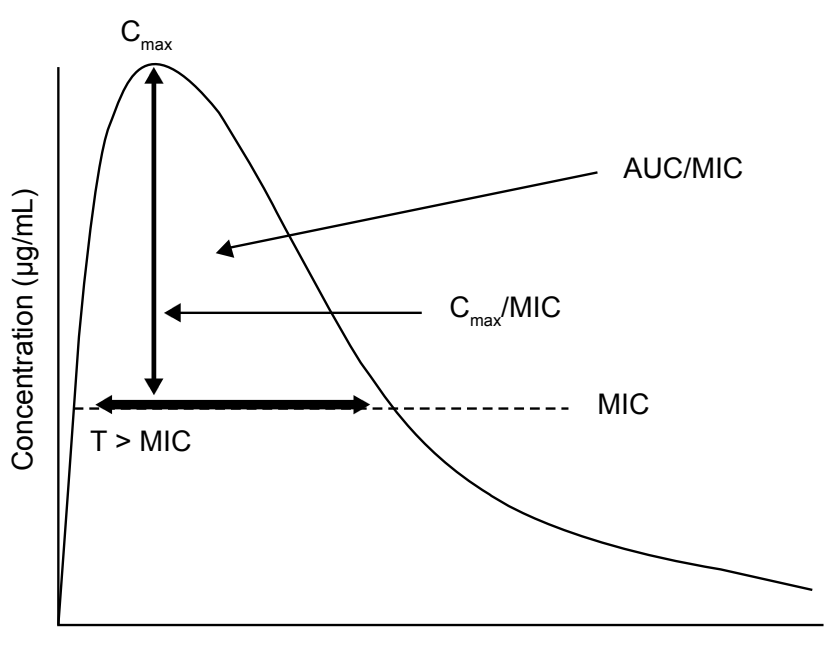

-

Figure 1: Pharmacokinetic / pharmacodynamic indices that describe antimicrobial effects. Cmax $=$ maximum concentration; $A U C / M I C=$ area under the curve / minimum inhibitory concentration; $\mathrm{T}>\mathrm{MIC}=$ time above the MIC.
$[21,22]$. Additionally, the $f \mathrm{~T}>\mathrm{MIC}$ exposure required for maximal killing of Gram-negative bacteria is often greater than that achieved for Gram-positives [22]. For example, the newest member of the carbapenem class, doripenem, required $27.3 \% \pm 11.7 \% f \mathrm{~T}>\mathrm{MIC}$ to achieve a 2-log reduction against Streptococcus pneumoniae, 35.4\% $\pm 5.0 \% f \mathrm{~T}>\mathrm{MIC}$ against $S$. aureus and $43.7 \% \pm 7.1 \%$ against Gramnegatives when tested in the classic murine in vivo thigh infection model [24]. The goal for this class of antibiotics is then to achieve the greatest amount of time during a dosing interval in order to achieve free drug concentrations above the MIC. This can be accomplished using several methods clinically [25]. The dosage of the antibiotic can be increased or more doses can be administered over a 24 hour period (i.e., shorten the dosing interval). Both of these methods increase T>MIC but does so inefficiently. Alternatively, the infusion duration for $\beta$-lactams can be increased, either by administering the entire daily dose continuously over a 24 hour period (i.e., continuous infusion), or by increasing the infusion duration enough to maximize the likelihood of achieving the pharmacodynamic target and the repeating this using the standard dosing interval. This latter concept, referred to as prolonged or extended-infusion $\beta$-lactam therapy has become quite popular over the recent decade due to its ability to increase the $f \mathrm{~T}>\mathrm{MIC}$ efficiently, while still providing an antibiotic free interval to administer other agents. These concepts will be discussed in greater detail later in this review.

In contrast to $\beta$-lactams, other time-dependent antibiotics can have persistent effects and when combined with small MIC ranges for pathogenic bacteria, the pharmacodynamic parameter best correlated with outcome becomes the AUC/MIC ratio. For example, although vancomycin is considered a time-dependent antimicrobial agent and trough concentrations are often analyzed clinically for therapeutic drug monitoring, it exhibits maximal bactericidal activity against S. aureus when the AUC/MIC (total drug) ratio is $\geq 400[26,27]$. A trough of $15-20 \mu \mathrm{g} / \mathrm{mL}$ correlates with this AUC/MIC exposure when the MIC is $1 \mu \mathrm{g} / \mathrm{mL}$ of less; therefore, therapeutic drug monitoring by use of trough concentrations are clinically useful for predicting pharmacodynamic exposure attainment [27]. For tigecycline, a timedependent glyclcycline antibiotic, the fAUC/MIC ratio is also most predictive of efficacy against $S$. aureus and Enterobacteriaceae $[28,29]$. The dosing strategy for these time-dependent antibiotics is to provide an overall daily dose that achieves the requisite AUC/MIC ratio without decreasing tolerability or increasing adverse events. For newer antibiotic agents such as tigecycline, the approved dosing regimen has often been selected with an understanding of pharmacodynamic requirements for common susceptible bacteria [23]. However, infections such as hospital acquired pneumonia, or bacteria such as $A$. baumannii, for which the drug is not approved, may require higher dosing regimens due to different pharmacodynamic targets, elevated MICs against the pathogen, or penetration to the site of infection. $[30,31]$. In contrast, concentration-dependent killing antibiotics achieve more rapid and greater antimicrobial effects when the free peak concentration is maximized in relationship with the MIC [21]. The pharmacodynamic parameters predictive of efficacy for these agents are the $f C_{\max } / \mathrm{MIC}$, the $f \mathrm{AUC} / \mathrm{MIC}$, or both. At clinically relevant concentrations, concentration-dependent killing antibiotics include the aminoglycosides, fluoroquinolones, daptomycin, polymyxin antibiotics, and metronidazole. Aminoglycosides are considered the classic concentration-dependent killing antibiotic class and achieve maximal bactericidal activity when their $\mathrm{fC}_{\max } / \mathrm{MIC}$ is greater than 10-12 [32,33]. A total drug AUC/MIC of at least 156 was also highly predictive of temperature resolution by day 7 for patients with hospital 
acquired pneumonia [33]. Therefore, clinical dosing regimens to optimize pharmacodynamics should attempt to maximize the $f \mathrm{C}_{\max }$ / MIC or AUC/MIC by increasing the individual dose and extending the dosing interval to prevent accumulation. Fluoroquinolone antibiotics are also concentration dependent, and the pharmacodynamic parameter best correlated with efficacy is an fCmax/MIC ratio of 12 or AUC/MIC (total drug) of 100-125 against Gram-negative bacteria, or a fAUC/MIC ratio of at least 30-50 against gram-positive bacteria such as S. pneumoniae [34-36]. More recently, the pharmacodynamics of the older polymyxin class of antibiotics has been found to observe concentration-dependent killing, where the $f A U C / M I C$ ratio is predictive of antibacterial effect against Gram-negative bacteria [37]. in vitro and in vivo thigh infection studies indicate that the $f$ AUC/MIC required for 1 - to $2-\log$ reductions against $P$. aeruginosa range from 15.6 to 22.8 and from 27.6 to 36.1 , respectively; in the lung infection model, those requirements ranged from 12.2 to 16.7 and 36.9 to 45.9 , respectively [38]. Similar targets were required for 1 -log reduction against $A$. baumannii isolates, although more variability was noted in the lung infection models [37].

\section{Optimizing Antibiotics}

Using the above knowledge, novel dosing strategies can be proposed to optimize the treatment of serious infections in hospitalized patients. Table 1 provides a list of common antibiotic classes, their pharmacodynamic parameter and target exposure, and strategies to optimize dosing for use in clinical practice. Of note, most of the dosing strategies discussed below are not approved by the Food and Drug Administration (FDA), as the science of pharmacodynamics has evolved greatly since the approval of many older antibiotics. Fortunately, many newer antibiotics today are designed with an understanding of pharmacodynamic principles and dosage regimens are selected based on the ability to optimize exposure against pathogens of interest for approved indications.

\section{Aminoglycosides}

Traditional or conventional dosing regimens of aminoglycosides call for two to three divided daily doses administered daily at doses of 1 to $1.5 \mathrm{mg} / \mathrm{kg}$ (gentamicin and tobramycin) or $7.5 \mathrm{mg} / \mathrm{kg}$ (amikacin) $[39,40]$. This method of dosing results in peak concentrations well below that needed to achieve the requisite pharmacodynamic thresholds against current bacteria and has notoriously been associated with nephro- and oto-toxicity due to higher average concentrations over a 24 hour period [41]. The alternative dosing regimen to this is known as high-dose, extended-interval or high-dose, once-daily aminoglycoside therapy.

Using a fixed $7 \mathrm{mg} / \mathrm{kg}$ intravenous dose of gentamicin and a dosing interval nomogram based on estimated creatinine clearance of patients, Nicolau et al. evaluated a once daily aminoglycoside dosing algorithm (referred to as the Hartford Nomogram) in 2,184 adult patients [42]. The investigators observed similar clinical response rates to historical data, but a reduced incidence of nephrotoxicity, which was $1.2 \%$ while using the Hartford Nomogram versus 3-5\% historically. Notably, the $7 \mathrm{mg} / \mathrm{kg}$ dose selected was based on several of our institution specific factors. The $\mathrm{MIC}_{90}$ for gentamicin against $P$. aeruginosa was $2 \mathrm{mcg} /$ $\mathrm{ml}$ in the hospital, and based on a population pharmacokinetic model from our patients, a $7 \mathrm{mg} / \mathrm{kg}$ daily dose was required to achieve an average peak of $20 \mathrm{mcg} / \mathrm{ml}$, approximately 10 times the MIC. Today, tobramycin is employed instead of gentamicin at our hospital; because it's $\mathrm{MIC}_{90}$ for $P$. aeruginosa remains about 2 MIC dilutions lower than gentamicin and still enables use of the $7 \mathrm{mg} / \mathrm{kg}$ dosing algorithm.

Therefore, not all hospital's employing a once daily aminoglycoside dosing strategy requires use of a $7 \mathrm{mg} / \mathrm{kg}$ daily dose. Hospitals with lower gentamicin or tobramycin MICs against $P$. aeruginosa may be able to use lower dosing regimens to achieve the pharmacodynamic threshold. Conversely, larger doses would be needed to treat MICs higher than $2 \mathrm{mcg} / \mathrm{ml}$. A pharmacokinetic/pharmacodynamics

\begin{tabular}{|c|c|c|c|}
\hline Antibiotic class & $\begin{array}{l}\text { Pharmacodynamic } \\
\text { (PD) profile }\end{array}$ & PD parameter & Clinical optimization strategy \\
\hline Aminoglyosides & $\begin{array}{l}\text { concentration- } \\
\text { dependent }\end{array}$ & $\begin{array}{l}f C \max / \mathrm{MIC} \geq 10 \text { to } 12 \\
\text { total } \mathrm{AUC} / \mathrm{MIC} \geq 156\end{array}$ & $\begin{array}{l}\text { High-dose, once-daily or extended interval dosing; dosing strategy can use a } \\
\text { nomogram (Hartford Nomogram) or be individualized using therapeutic drug } \\
\text { monitoring and MIC }\end{array}$ \\
\hline $\begin{array}{l}\text { B-lactams } \\
\text { penicillins } \\
\text { carbapenems } \\
\text { cephalosporins }\end{array}$ & $\begin{array}{l}\text { time-dependent } \\
\text { time-dependent } \\
\text { time-dependent }\end{array}$ & $\begin{array}{l}f \mathrm{~T}>\mathrm{MIC} \geq 50 \\
f \mathrm{~T}>\mathrm{MIC} \geq 30-40 \\
f \mathrm{~T}>\mathrm{MIC} \geq 50-70\end{array}$ & $\begin{array}{l}\text { Continuous or prolonged infusion; can be combined with greater doses to treat higher } \\
\text { MIC organisms }\end{array}$ \\
\hline Fluoroquinolones & $\begin{array}{l}\text { concentration- } \\
\text { dependent }\end{array}$ & $\begin{array}{c}f C \text { max/MIC } \geq 10 \text { to } 12 ; \\
\text { total AUC/MIC }>125 \text { for } \\
\text { gram-negatives; } f \text { AUC/ } \\
\text { MIC }>30-50 \text { for gram- } \\
\text { positives }\end{array}$ & $\begin{array}{l}\text { Increase dose related to MIC; however careful of increases in toxicity associated with } \\
\text { higher concentrations; Use the most potent agent (i.e., lowest MIC) to maximize AUC/ } \\
\text { MIC ratio }\end{array}$ \\
\hline $\begin{array}{l}\text { Glycopeptides/Lipopeptides } \\
\text { daptomycin } \\
\text { vancomycin }\end{array}$ & $\begin{array}{l}\text { concentration- } \\
\text { dependent } \\
\text { time-dependent }\end{array}$ & $\begin{array}{l}\text { fAUC/MIC; } f \mathrm{Cmax} / \mathrm{MIC} \text { * } \\
\text { total AUC/MIC }>400\end{array}$ & $\begin{array}{l}\text { Maximize dose in relation to MIC } \\
\text { Maximize over daily dose in relation to MIC; target trough concentrations of } 15-20 \\
\mathrm{mcg} / \mathrm{ml}\end{array}$ \\
\hline Macrolides/Azalides & time-dependent & $\mathrm{AUC} / \mathrm{MIC}$ * & $\mathrm{N} / \mathrm{A}$ \\
\hline Oxazolidinone (linezolid) & time-dependent & total AUC/MIC > 110 & $\begin{array}{l}\text { Maximize overall daily dose in relation to MIC; standard dose optimized for most } \\
\text { susceptible bacteria up to MIC of } 2 \mathrm{mcg} / \mathrm{ml} .\end{array}$ \\
\hline Polymyxins & $\begin{array}{l}\text { concentration- } \\
\text { dependent }\end{array}$ & $\begin{array}{l}\text { fAUC/MIC > } 12 \text { to } 15 \\
\text { total } \mathrm{AUC} / \mathrm{MIC}>60\end{array}$ & $\begin{array}{l}\text { Maximize overall daily dose in relation to MIC while considering nephrotoxicity; } \\
\text { Consider algorithm for loading and maintenance doses by Garonzik (70) }\end{array}$ \\
\hline $\begin{array}{l}\text { Tetracyclines/Glycylcyclines } \\
\text { doxycycline } \\
\text { tigecycline }\end{array}$ & $\begin{array}{l}\text { time-dependent } \\
\text { time-dependent }\end{array}$ & $\begin{array}{l}\text { AUC/MIC * } \\
\text { fAUC/MIC }\end{array}$ & $\begin{array}{l}\text { N/A } \\
\text { Approved dosage optimized for most susceptible bacteria in intra-abdominal infections } \\
\text { and complicated skin infections; if tolerated, increase overall daily dose to } 200 \mathrm{mg} \text { daily } \\
\text { to maximize pharmacodynamics for more serious infections or Acinetobacter spp. }\end{array}$ \\
\hline
\end{tabular}

*Clinically relevant AUC/MIC targets for these antibiotics have not been well established

Table 1: Summary of antibiotic classes, pharmacodynamics parameter, exposure threshold and strategy to optimize pharmacodynamics 
simulation study recently conducted by Drusano and Louie observed that the probability of day 7 temperature resolution using a $10 \mathrm{mg} / \mathrm{kg}$ daily dose against an organism with an MIC of $4 \mathrm{mcg} / \mathrm{ml}$ was $79.7 \%$, with an estimated probability of nephrotoxicity well below 1\% [41]. In contrast, a $5 \mathrm{mg} / \mathrm{kg}$ dose administered every 12 hours provided a $53.6 \%$ likelihood of effect against an MIC of $4 \mathrm{mcg} / \mathrm{ml}$, with a $24.6 \%$ likelihood of nephrotoxicity. Additionally, patient populations with altered pharmacokinetics may require lower or larger doses depending on individual clearance or volume of distribution, as well as MIC. In a study by Rea et al. in 102 critically ill patients, due to a larger volume of distribution [mean of $53 \mathrm{~L}$ (95\% confidence interval: 38-67 L)], a 7 $\mathrm{mg} / \mathrm{kg}$ dose in the investigator's population had a $10 \%$ likelihood of achieving a $\mathrm{C}_{\text {a }} / \mathrm{MIC}$ of 10 against $P$. aeruginosa with an MIC of 2 $\mathrm{mcg} / \mathrm{ml}$ [43]. This likelihood increased to $50 \%$ and $88 \%$ at MICs of 1 $\mathrm{mcg} / \mathrm{ml}$ and $0.5 \mathrm{mcg} / \mathrm{ml}$ respectively. Conflicting literature exists as to whether critically ill patients require larger doses of aminoglycosides then $7 \mathrm{mg} / \mathrm{kg}$ to achieve the threshold $f \mathrm{C}_{\max } / \mathrm{MIC}$ ratio of at least 10 [44-47].

An alternate approach to a nomogram designed dose is to employ individualized dosing in specific patients based on the results of multiple serum concentrations, the resulting or estimated peak concentration and the MIC of the pathogen [48,49]. Although this provides a highly individualized and accurate approach, this may be time-consuming and burdensome in some hospitals if applied to all patients. Additionally, the MIC of the pathogen is the greatest factor in this equation since it lies in the denominator of the pharmacodynamic equation $\left(f \mathrm{C}_{\max } /\right.$ MIC or $f$ AUC/MIC). Thus one-dilution changes in the MIC result in doubling or halving of the pharmacodynamics exposure. Indeed, for isolates with lower MICs (below $2 \mathrm{mcg} / \mathrm{ml}$ ), the nomogram approach is very reliable in obtaining the requisite exposure, while greater MICs and more variable pharmacokinetic profiles (i.e., variations in renal function) may benefit from a more individualized approach.

Regardless of which approach to high-dose, once-daily or extendedinterval dosing of aminoglycoside is employed, this pharmacodynamic dosing strategy is today the standard of practice for aminoglycoside dosing. In a national survey of 500 acute care hospitals, 3 of every 4 hospitals have now adopted the use of this strategy which correlates to a 4-fold increase in use since 1993, when a similar survey of 336 acute care hospitals resulted in a mere 19\% reporting the use of pharmacodynamics based dosing [50].

\section{$\beta$-lactams}

Traditional dosing for $\beta$-lactam antibiotics typically involves administering smaller doses over 15-30 minute infusions 2-4 times daily, depending on the serum half-life of the antibiotic and the patient's kidney. This strategy often does not achieve the requisite $f \mathrm{~T}>\mathrm{MIC}$ thresholds for all susceptible bacteria. DeRyke et al. used Monte Carlo simulation to arrive at a likelihood of achieving $f \mathrm{~T}>\mathrm{MIC}$ targets using current susceptibility breakpoints versus that of pharmacodynamics derived breakpoints for $7 \beta$-lactams [51]. As demonstrated in table 2 , susceptibility breakpoints are traditionally set for a single dosing regimen against an organism, while pharmacodynamics breakpoints are dosing regimen specific. A higher susceptibility breakpoint (i.e., more organisms in the population would be defined as susceptible and considered 'treatable') can be justified if larger doses administered more frequently were employed. Importantly, standard clinical doses for most of the tested $\beta$-lactams were unable to achieve the requisite $f \mathrm{~T}>\mathrm{MIC}$ threshold at the susceptibility breakpoint, as noted by lower pharmacodynamics breakpoints for these regimens. For example, a standard cefepime regimen of $2 \mathrm{~g}$ every 12 hours administered as a 30

\begin{tabular}{|l|c|c|}
\hline \multirow{2}{*}{$\begin{array}{c}\text { Drug regimen (all 30 minute } \\
\text { infusions) }\end{array}$} & \multicolumn{2}{|c|}{ Susceptibility breakpoint MIC (mcg/ml) } \\
\cline { 2 - 3 } & CLSI & Pharmacodynamic \\
\hline Cefepime 1 g q12 h & 8 & 2 \\
\hline Cefepime 2 g q12 h & 8 & 4 \\
\hline Cefepime 2 g q8 h & 8 & 16 \\
\hline Ceftazidime 1 g q8 h & 8 & 8 \\
\hline Ceftazidime 2 g q8 h & 8 & 16 \\
\hline Imipenem 1 g q8 h & $4^{a}$ & 2 \\
\hline Meropenem 1 g q8 h & $4^{a}$ & 2 \\
\hline Piperacillin/tazobactam 3.375 g q6 h & $64^{b}$ & 8 \\
\hline Piperacillin/tazobactam 4.5 g q6 h & $64^{b}$ & 8 \\
\hline
\end{tabular}

${ }^{a} \mathrm{CLSI}$ susceptibility breakpoints for imipenem and meropenem against $P$. aeruginosa were reduced to $2 \mathrm{mcg} / \mathrm{ml}$ in 2012

${ }^{b} \mathrm{CLSI}$ susceptibility breakpoints for piperacillin/tazobactam against $P$. aeruginosa were reduced to $16 \mathrm{mcg} / \mathrm{ml}$ in 2012

Table 2: Clinical Laboratory Standards Institute (CLSI) susceptibility breakpoints for several $\tilde{\beta}$ lactam antibiotics against Pseudomonas aeruginosa versus pharmacodynamics derived breakpoints, which are based on the ability for a dosing regimen to attain the $f \mathrm{~T}>\mathrm{MIC}$ exposure threshold for all bacteria defined as susceptible. Derived from DeRyke and colleagues [51]

minute infusion achieves a high likelihood of achieving 50\% $f \mathrm{~T}>\mathrm{MIC}$ only up to a MIC of $4 \mathrm{mcg} / \mathrm{ml}$, while $P$. aeruginosa with a MIC of $8 \mathrm{mcg} /$ $\mathrm{ml}$ would still be considered susceptible. This puts patients receiving these standard regimens against organisms with MICs at or near the breakpoint at risk for clinical failure or the development of resistance because the $f \mathrm{~T}>\mathrm{MIC}$ threshold may not have been achieved clinically. The solution to such a scenario is to either reduce the susceptibility breakpoint in line with these data, as was recently done for piperacillin/ tazobactam and $P$. aeruginosa (i.e., the breakpoint is now $16 \mathrm{mcg} / \mathrm{ml}$ instead of $64 \mathrm{mcg} / \mathrm{ml}$ ), or modify the dosing strategy to increase the likelihood of obtaining $f \mathrm{~T}>\mathrm{MIC}$ against higher MIC organisms.

Various methods have been employed to optimize the pharmacodynamics of the $\beta$-lactams, including giving higher dosages, administering the drugs more often, and prolonging the infusion time (either to 3-4 hours depending on room temperature stability or continuously over 24 hours). In general, the most effective way to optimize these agents, particularly for higher MIC organisms, is to both increase the administered dose and prolong the infusion, thereby maintaining a concentration above higher MICs for the required bactericidal exposure time. This has been applied to $\beta$-lactams such as cefepime and meropenem in clinical studies $[52,53]$ Dosages of 2 grams every 8 hours (each dose administered as a 3 hour prolonged infusion) in patients with normal kidney function achieve a high probability of treating organisms considered resistant with MICs of $16 \mu \mathrm{g} / \mathrm{ml}$ and 32 $\mu \mathrm{g} / \mathrm{ml}$ for meropenem and cefepime, respectively, which is significantly greater than if the same dosage regimen were infused over the standard 30 minutes.

Perhaps the most common $\beta$-lactam that these concepts have been applied to clinically is piperacillin/tazobactam. Both continuous and prolonged infusion techniques have been evaluated to optimize pharmacodynamics in several studies [54-57]. Using Monte Carlo simulation, Kim et al. found that a $4.5 \mathrm{~g}$ every 6 hour dose (with each dose infused over 3 hours) would achieve a similar pharmacodynamic exposure to the same daily dose $(18.0 \mathrm{~g})$ administered as a continuous infusion, and both would have higher probabilities of target attainment than the standard $4.5 \mathrm{~g}$ every 6 hour (30 minute infusion) dose at MICs ranging from $16 \mathrm{mcg} / \mathrm{ml}$ to $64 \mathrm{mcg} / \mathrm{ml}$ [55]. Importantly, the likelihood of achieving $50 \% f \mathrm{~T}>\mathrm{MIC}$ at a MIC of $64 \mathrm{mcg} / \mathrm{ml}$ remained less than $30 \%$ for these regimens, further justifying the reduction in the CLSI susceptibility breakpoint to $16 \mathrm{mcg} / \mathrm{ml}$ for $P$. aeruginosa. 
Clinically, continuous infusion piperacillin/tazobactam has been employed at the authors' institution since 1999 with good success using dosages ranging from 9 grams daily to 18 grams daily depending on the type of infection (e.g., higher dosages for pneumonia and bacteremia versus intra-abdominal and complicated skin and skin structure infections) and the patient's kidney function [54]. Benefits of a continuous infusion modality for piperacillin/tazobactam include its once daily administration, a reduction in daily dose for less severe infections leading to lower drug costs, and optimal time above the MIC. However, a 24 hour continuous infusion does require a dedicated intravenous site if other drugs with compatibility issues also require co-administration.

Circa 2005, Lodise et al. implemented a piperacillin/tazobactam dosing regimen at their medical center whereby all piperacillin/ tazobactam orders were changed to 3.375 g every 8 hours (4 hour prolonged infusions) and compared the mortality and length of stay of this regimen with a historic control of patients who received $3.375 \mathrm{~g}$ every 6 hours (as 30 minute infusion) for $P$. aeruginosa infections [56]. Patients receiving the prolonged infusion had a lower 14-day mortality rate $(12.2 \% v s .31 .6 \%, p=0.04)$ and shorter hospital stay (21 days vs. 38 days, $p=0.02)$ that reached statistical significance when limited to critically-ill patients with an APACHE II score of $\geq 17$. These data confirm that, like continuous infusion, prolonged infusion is also a viable option for maximizing the outcomes of piperacillin/tazobactam against $P$. aeruginosa, and can do so while permitting a 4 hour window each dosing interval to administer other intravenous medications. However, according to pharmacodynamic models, the $3.375 \mathrm{~g}$ every 8 hour ( 4 hour infusion) regimen would be inadequate for isolates with MICs $\geq 32 \mu \mathrm{g} / \mathrm{mL}$, thus it would be beneficial to know the piperacillin/ tazobactam MIC distribution of the $P$. aeruginosa population in one's institution before implementing this dosage regimen [55]. Similarly, in a multicenter, retrospective medical record review, the RECEIPT Study evaluated 359 adult patients treated for gram-negative infections with either a 4-hour extended-infusion piperacillin-tazobactam $(n=186)$ or non-extended-infusions of cefepime, ceftazidime, imipenem-cilastatin, meropenem, doripenem, or piperacillin-tazobactam $(n=173)$. The primary outcome measured was mortality rate between the two groups respectively with secondary outcomes of hospital length of stay, ICU length of stay, and total duration of antibiotic therapy. Although baseline characteristics of the cohorts were slightly different, the investigators observed similar rates of antibiotic duration, hospital length of stay, and ICU length of stay, but in-hospital mortality was significantly less in the extended-infusion piperacillin-tazobactam group versus the comparators $(9.7 \%$ vs. $17.9 \%, \mathrm{p}=0.02)$. A multivariate analysis revealed the extended infusion group prolonged survival by 2.77 days $(\mathrm{p}<0.01)$ and reduced the risk of mortality (odds ratio 0.43 , $\mathrm{p}=0.05)$ [57].

Recently, a systematic review and meta-analysis of observation studies assessing the clinical outcomes of extended or continuous infusion carbapenems or piperacillin/tazobactam versus standard infusion demonstrated a significant advantage to this pharmacodynamics strategy [58]. Mortality was lower among patients receiving extended or continuous infusion [relative risk (RR), 0.59; $95 \%$ confidence interval (CI), 0.41-0.83]. This was most notable among patients with pneumonia (RR, 0.50; 95\% CI, 0.26-0.96) suggesting these dosage strategies may provide the greatest benefits for critically ill patients, or patients likely to be infected with more resistant organisms. Indeed, in the same issue of Clinical Infectious Diseases, investigators from Australia and Hong Kong presented the first prospective, double-blind, randomized controlled trial of continuous versus standard infusion $\beta$-lactam therapy in patients with severe sepsis [59]. Sixty patients from 5 different intensive care units were randomized to receive piperacillin/tazobactam, meropenem, or ticarcillin/ clavulanate as continuous or standard 30 minute infusion regimens. The investigators observed plasma concentrations for the continuous infusion cohort to exceed the MIC in $82 \%$ of patients versus only $29 \%$ of the standard intermittent infusion cohort $(\mathrm{p}=0.001)$. Clinical cure was also higher in the continuous infusion group (70\% vs. $43 \%$, $\mathrm{p}=0.037$ ); however, there was no observed difference in survival These observations are similar to other small non-randomized studies that reported greater clinical success rates with continuous infusion piperacillin/tazobactam, meropenem, and ceftriaxone versus that of standard infusion in critically ill patients [60-62]. Finally, a doubleblind, randomized controlled study of doripenem $500 \mathrm{mg}$ q8h as a 4 hour prolonged infusion versus standard infusion imipenem-cilastatin in patients with ventilator-associated pneumonia demonstrated noninferiority between dosing regimens; however, clinical success against the small population of patients infected with $P$. aeruginosa was numerically in favor of the prolonged infusion regimen $[80 \%(16 / 20)$ vs. 43\% (6/14)] [63]. Unfortunately, these results could not be replicated in a randomized controlled trial evaluating shorter courses of a higher dose prolonged infusion doripenem [64].

Collectively, these data suggest that continuous or prolonged infusion dosing strategies are effective in optimizing $\beta$-lactam pharmacodynamics and may result in a clinically superior dosing modality compared with standard infusion, particularly for critically ill patients or those infected with higher MIC pathogens. Selection of which antibiotic to apply these strategies to and at what dose depends on several factors, one of which is drug stability. Most beta-lactam antibiotics are stable for at least 24 hours at room temperature, and thus can be administered as a 24 hour continuous infusion or as prolonged infusion. However, certain beta-lactams including the carbapenems (imipenem, meropenem, doripenem), ampicillin, and the newest cephalosporin, ceftaroline, are not stable at room temperature for a full 24 hours. As a result, these antibiotics are better suited for administration as a prolonged infusion to enhance pharmacodynamic exposure while retaining room temperature stability requirements. Additional factors include the available antibiotics in one's hospital, the severity of illness of patients to receive these agents, and most importantly, the MIC distribution of target pathogens.

For example, the authors employed a strategic process to identify the most likely pathogens causing ventilator associated pneumonia in their intensive care units (ICUs), the MIC distributions for the most common Gram-negative (i.e., P. aeruginosa), and pharmacodynamics modeling to select the antibiotic and dosing regimen to empirically treat patients [65]. Due to substantial differences in MIC distributions for $P$. aeruginosa between the three included ICUs, different antibiotic regimens were implemented as empiric therapy. These included prolonged infusion regimens of meropenem ( $2 \mathrm{~g}$ q8h, $3 \mathrm{~h}$ infusion), cefepime ( $2 \mathrm{~g} \mathrm{q} 8 \mathrm{~h}, 3 \mathrm{~h}$ infusion), and piperacillin/tazobactam (18 g continuous infusion) for patients with normal kidney function. After one year of implementing this program, appropriate antibiotic therapy was significantly increased in the intervention cohort and infectionrelated mortality was reduced by $69 \%(8.5 \%$ vs. $21.6 \%, \mathrm{p}=0.029)$.

\section{Clinical Pharmacodynamics of Other Antibiotic Classes}

We refer the reader to table 1 for pharmacodynamic strategies for various other antibiotic classes. For newer antibiotic agents (i.e., those approved over the last decade), pharmacodynamics has typically 
been considered in dosing regimen selection. As a result, for most susceptible bacteria listed in the approved indications, these antibiotics at approved dosages (e.g., linezolid $600 \mathrm{mg}$ q12h, daptomycin 4-6 mg/ $\mathrm{kg}$ q24h, tigecyline $50 \mathrm{mg}$ q12h, etc.) have a high likelihood of obtaining pharmacodynamic exposure thresholds. We will, therefore, focus our remaining discussion on older antibiotic classes where standard dosing regimens likely did not consider pharmacodynamic theory.

\section{Fluoroquinolones}

While fluoroquinolone antibiotics are widely utilized and listed in the guidelines for the treatment of various infections (pneumonia, community and hospital-acquired; urinary tract infections; etc.), the poor susceptibilities for many gram-negative organisms that potentially cause these infections should make them second line considerations [20]. From a pharmacodynamic perspective, fluoroquinolones are unable to achieve bactericidal exposure (AUC/MIC > 125) at standard dosages for not only bacteria considered resistant, but also a number of bacteria that the microbiology laboratory would classify as susceptible [51]. This is a result of a higher than acceptable breakpoint used to define susceptibility for Gram-negatives $(\leq 1 \mu \mathrm{g} / \mathrm{ml}$ for ciprofloxacin and $\leq 2 \mu \mathrm{g} / \mathrm{ml}$ for levofloxacin). Pharmacodynamic simulation studies suggest the proper breakpoints should be $0.25 \mu \mathrm{g} / \mathrm{ml}$ and $0.5 \mu \mathrm{g} / \mathrm{ml}$, respectively, which would significantly increase resistance rates further at most hospitals. As a result, ciprofloxacin $400 \mathrm{mg}$ every 8 hour and levofloxacin $750 \mathrm{mg}$ every 24 hour regimens historically have achieved low probabilities of attaining the required pharmacodynamic exposure against $P$. aeruginosa isolates, as well as for the empiric treatment of hospital acquired pneumonia $[66,67]$. The pharmacodynamic strategy for fluoroquinolones against Gram-negatives is to; therefore, maximize the overall daily exposure through dosage increases in relation to the MIC. However, fluoroquinolones may not be tolerated at higher doses because peak concentrations are related to central nervous stimulation and gastrointestinal side effects. Thus the optimal fluoroquinolone dosing regimen is the maximum tolerated dose of the agent with the lowest MIC, so as to maximize AUC/MIC. If this exposure threshold cannot be obtained, these agents should be reserved for combination therapy regimens.

Against S. pneumoniae causing community acquired respiratory tract infections; a fAUC/MIC of at least 33.7 was required to optimize clinical response. Levofloxacin (intravenous and oral), moxifloxacin (intravenous and oral), and gemifloxacin (oral only) are currently the only available fluoroquinolones in the United States with sufficient microbiological activity against $S$. pneumoniae. Currently, resistance to these 'respiratory' fluoroquinolones is rare and standard dosage regimens (levofloxacin $750 \mathrm{mg}$ once daily, moxifloxacin $400 \mathrm{mg}$ once daily, and gemifloxacin $320 \mathrm{mg}$ once daily) provide a high likelihood of achieving $f$ AUC/MIC ratios of at least 30 against this organism in most patients $[68,69]$.

\section{Polymyxins}

The polymyxin class of antibiotics includes polymyxin B and polymyxin E (colistin), the latter of which is administered clinically as colistin methane sulfate intravenously. Although an older class of antibiotics, their use has been revitalized by the multi-drug and panresistant strains of $P$. aeruginosa, A. baumannii, and carbapenemresistant Enterobacteriaceae that has arisen over the last decade [37]. Notably, these agents were shelved in the early 1970's because of high rates of nephrotoxicity and neurotoxicity relative to other available antibiotic agents. Their pharmacokinetic/pharmacodynamic properties have only recently begun to be elucidated, and antimicrobial effect is correlated with fAUC/MIC exposures. To date, these experiments have largely been undertaken in vitro or in animal infection models. As previously mentioned, $1-\log$ reductions in colony forming unit require fAUC/MIC ratios of approximately 12.2 to 22.8 in both thigh- and lung-infection murine models [37]. Unfortunately, the protein binding of colistin in humans, particularly critically ill patients, has not been established; therefore, for dosing strategies, a total AUC/MIC ratio of approximately 60 has been used to target drug concentrations [70]. Garonzik et al. used a population pharmacokinetic model from 105 critically ill patients receiving various clinical doses of colistin methane sulfate to derive recommendations for loading and maintenance doses. We refer the reader to the original reference for details on the weightbased, MIC-based dosing algorithm [70]. The investigators note, however, that because colistin methane sulfate, which is eliminated in the kidney, must be converted to active colistin for microbiological activity, and therefore obtaining therapeutic concentrations needed to achieve the AUC/MIC target would be unlikely in patients with a creatinine clearance $(\mathrm{CrCL})$ greater than $70 \mathrm{ml} / \mathrm{min}$ or against an MIC greater than $0.5 \mathrm{mcg} / \mathrm{ml}$, without increasing risk for nephrotoxicity. Based on pharmacokinetic and pharmacodynamic data, the most reasonable approach for polymyxins is to use them as part of a highly active antibiotic combination.

\section{Vancomycin}

Vancomycin, a glycopeptide, has long been considered a time-dependent killing antibiotic, and therefore therapeutic drug concentration monitoring by using peak concentrations, troughs, or both, has been practiced for years. Therapeutic drug monitoring for vancomycin has also been largely debated for the same amount of time. [71]. Current consensus for treatment of serious S. aureus infections is that vancomycin is a slowly-cidal, time-dependent killing antibiotic [72]. However, AUC/MIC, not T $>$ MIC, is the most predictive pharmacodynamic parameter $[26,27]$. This concept, unfortunately, is directly opposed to guidelines that indicate that a trough of $15-20$ $\mathrm{mcg} / \mathrm{ml}$ is sufficient for therapeutic drug monitoring of the drug [72] First, a trough alone is insufficient to estimate a patient's 24 hour AUC, unless incorporated into a population pharmacokinetic model using a Bayesian approach. Even so, an additional, often a peak, concentration is required to generate a more accurate estimate of clearance and AUC. Second, not all patients who obtain troughs of $15-20 \mathrm{mcg} / \mathrm{ml}$ obtain 24 hour AUCs sufficient to achieve an AUC/MIC of at least 400 [73]. Finally, the MIC is once again in the denominator for this pharmacodynamic parameter, and a single dilution in each direction will affect exposure significantly. Currently, standard dosing regimens of vancomycin aimed at obtaining troughs of $15-20 \mathrm{mcg} / \mathrm{ml}$ are not adequate to achieve the target AUC/MIC of 400 when the vancomycin MIC is above $1 \mathrm{mcg} / \mathrm{ml}$ [73]. Thus, based on this model, S. aureus with a vancomycin MIC of $2 \mathrm{mcg} / \mathrm{ml}$ are considered 'untreatable', yet remain susceptible given the current CLSI breakpoints. Although numerous studies have linked higher MICs with clinical failure and mortality, many patients receiving vancomycin against organisms at an MIC of $2 \mathrm{mcg} / \mathrm{ml}$ do well [74]. It is also well known that higher doses of vancomycin (upwards of 4 grams per day) cause a significant increase in nephrotoxocity, and in some patient populations it is not possible to achieve a trough of $15-20 \mathrm{mcg} / \mathrm{ml}$ without using dosages that result in toxicity $[73,75]$. Despite years of use and clinical studies, the optimal dosing strategy for vancomycin that optimized kill of $S$. aureus while minimizing nephrotoxicity has yet to be established [76]. Until then, clinicians are advised to at the very least continue to aim for trough concentrations of $15-20 \mathrm{mcg} / \mathrm{ml}$, or collect sufficient information to calculate the AUC/MIC ratio, which would be several plasma concentrations and a MIC [72,73]. 


\section{Conclusions}

In the absence of new antibiotics to treat current pathogenic bacteria, the incorporation of pharmacodynamic principles into designing optimal dosing regimens for clinical practice is paramount. Knowledge of the relationship between antibiotic concentration to microbiological potency and its effect on antibacterial activity can lead to the design of dosing regimens that optimize killing of bacteria in the clinical setting. Fortunately, these optimal dosing strategies are now defined for many of the available antibiotics we use frequently, including aminoglycosides and $\beta$-lactams. Pharmacodynamic strategies for other antibiotic classes continue to evolve.

\section{References}

1. Klevens RM, Edwards JR, Richards CL Jr, Horan TC, Gaynes RP, et al. (2007) Estimating health care-associated infections and deaths in U.S. hospitals, 2002. Public Health Rep 122: 160-166.

2. Caron WP, Mousa SA (2010) Prevention strategies for antimicrobial resistance: a systematic review of the literature. Infect Drug Resist 3: 25-33.

3. Zell BL, Goldmann DA (2007) Healthcare-associated infection and antimicrobial resistance: moving beyond description to prevention. Infect Control Hosp Epidemiol 28: 261-264

4. Rice LB (2008) Federal funding for the study of antimicrobial resistance in nosocomial pathogens: no ESKAPE. J Infect Dis 197: 1079-1081.

5. Boucher HW, Talbot GH, Bradley JS, Edwards JE, Gilbert D, et al. (2009) Bad bugs, no drugs: no ESKAPE! An update from the Infectious Diseases Society of America. Clin Infect Dis 48: 1-12.

6. Klevins RM, Edwards JR, Tenover FC, McDonald LC, Horan T, et al. (2006) Changes in the epidemiology of methicillin-resistant Staphylococcus aureus in intensive care units in US hospitals, 1992-2003. Clin Infect Dis 42: 389-391.

7. Boucher HW, Corey GR (2008) Epidemiology of methicillin-resistant Staphylococcus aureus. Clin Infect Dis 46: S344-349.

8. Boucher HW (2010) Challenges in anti-infective development in the era of bad bugs, no drugs: a regulatory perspective using the example of bloodstream infection as an indication. Clin Infect Dis 1: S4-S9.

9. Hamad B (2010) The antibiotics market. Nat Rev Drug Discov 9: 675-676.

10. Payne DJ, Gwynn MN, Holmes DJ, Pompliano DL (2007) Drugs for bad bugs: confronting the challenges of antibacterial discovery. Nat Rev Drug Discov 6: 29-40.

11. Gwynn MN, Portnoy A, Rittenhouse SF, Payne DJ (2010) Challenges of antibacterial discovery revisited. Ann N Y Acad Sci 1213: 5-19.

12. Silver LL (2011) Challenges of antibacterial discovery. Clin Microbiol Rev 24 71-109.

13. (2010) The $10 \times$ ' 20 Initiative: pursuing a global commitment to develop 10 new antibacterial drugs by 2020. Clin Infect Dis 50: 1081-1083.

14. (2012) European Centre for Disease Prevention and Control/European Medicines Agency. European Centre for Disease Prevention and Control/ European Medicines Agency Joint Technical Report. The Bacterial Challenge: Time to React.

15. Butler MS, Cooper MA (2011) Antibiotics in the clinical pipeline in 2011. J Antibiot (Tokyo) 64: 413-425.

16. Jabes D (2011) The antibiotic R\&D pipeline: an update. Curr Opin Microbiol 14: $564-569$

17. Elligsen M, Walker SA, Simor A, Daneman N (2012) Prospective audit and feedback of antimicrobial stewardship in critical care: program implementation, experience, and challenges. Can J Hosp Pharm 65: 31-36.

18. Dellit TH, Owens RC, McGowan JE Jr, Gerding DN, Weinstein RA, et al (2007) Infectious Diseases Society of America and the Society for Healthcare Epidemiology of America guidelines for developing an institutional program to enhance antimicrobial stewardship. Clin Infect Dis 44: 159-177.

19. Doron S, Davidson LE (2011) Antimicrobial stewardship. Mayo Clin Proc 86 1113-1123.
20. Eagle H, Fleishchman R, Musselman AD (1950) Effect of schedule of administration on the therapeutic efficacy of penicillin; importance of the aggregate time penicillin remains at effectively bactericidal levels. Am J Med 9: 280-299.

21. Craig WA (1998) Pharmacokinetic/pharmacodynamic parameters: rationale for antibacterial dosing of mice and men. Clin Infect Dis 26: 1-10.

22. Turnidge JD (1998) The pharmacodynamics of beta-lactams. Clin Infect Dis 27: 10-22.

23. Ambrose PG, Bhavnani SM, Rubino CM, Louie A, Gumbo T, et al. Pharmacokinetics-pharmacodynamics of antimicrobial therapy: it's not just for mice anymore. Clin Infect Dis 44: 79-86.

24. Paterson DL, DePestel DD (2009) Doripenem. Clin Infect Dis 49: 291-298

25. Gillespie EL, Kuti JL, Nicolau DP (2005) Pharmacodynamics of antimicrobials treatment optimisation. Expert Opin Drug Metab Toxicol 1: 351-361.

26. Moise-Broder PA, Forrest A, Birmingham MC, Schentag JJ (2004) Pharmacodynamics of vancomycin and other antimicrobials in patients with Staphylococcus aureus lower respiratory tract infections. Clin Pharmacokinet 43: 925-942.

27. Kullar R, Davis SL, Levine DP, Rybak MJ (2011) Impact of vancomycin exposure on outcomes in patients with methicillin-resistant Staphylococcus aureus bacteremia: support for consensus guideline suggested targets. Clin Infect Dis 52: 975-981.

28. Nicasio AM, Crandon JL, Nicolau DP (2009) In vivo pharmacodynamic profile of tigecycline against phenotypically diverse Escherichia coli and Klebsiella pneumoniae isolates. Antimicrob Agents Chemother 53: 2756-2761.

29. Koomanachai P, Crandon JL, Banevicius MA, Peng L, Nicolau DP (2009) Pharmacodynamic profile of tigecycline against methicillin-resistan Staphylococcus aureus in an experimental pneumonia model. Antimicrob Agents Chemother 53: 5060-5063.

30. Bhavnani SM, Rubino CM, Hammel JP, Forrest A, Dartois N, et al. (2012 Pharmacological and patient-specific response determinants in patients with hospital-acquired pneumonia treated with tigecycline. Antimicrob Agents Chemother 56: 1065-72.

31. Koomanachai P, Kim A, Nicolau DP (2009) Pharmacodynamic evaluation of tigecycline against Acinetobacter baumannii in a murine pneumonia model. $J$ Antimicrob Chemother 63: 982-987.

32. Moore RD, Smith CR, Lietman PS (1984) Association of aminoglycoside plasma levels with therapeutic outcome in gram-negative pneumonia. Am J Med 77: 657-662.

33. Kasuba AD, Bertino JS Jr, Nafziger AN (1998) Dosing of aminoglycosides to rapidly attain pharmacodynamic goals and hasten therapeutic response by using individualized pharmacokinetic monitoring of patients with pneumonia caused by gram-negative organisms. Antimicrob Agents Chemother 42: 1842 1844.

34. Forrest A, Nix DE, Ballow CH, Goss TF, Birmingham MC, et al. (1993) Pharmacodynamics of intravenous ciprofloxacin in seriously ill patients. Antimicrob Agents Chemother 37: 1073-1081.

35. Preston SL, Drusano GL, Berman AL, Fowler CL, Chow AT, et al. (1998) Pharmacodynamics of levofloxacin: a new paradigm for early clinical trials. JAMA 279: 125-129.

36. Ambrose PG, Grasela DM, Grasela TH, Passarell J, Mayer HB, et al. (2001) Pharmacodynamics of fluoroquinolones against Streptococcus pneumoniae in patients with community-acquired respiratory tract infections. Antimicrob Agents Chemother 45: 2793-2797.

37. Bergen PJ, Landersdorfer CB, Zhang J, Zhao M, Lee HJ, et al. (2012) Pharmacokinetics and pharmacodynamics of 'old' polymyxins: what is new? Diagn Microbiol Infect Dis 74: 213-223.

38. Dudhani RV, Turnidge JD, Coulthard K, Milne RW, Ranyer CR, et al. (2010) Elucidation of the pharmacokinetic/pharmacodynamic determinant of colistin activity against $P$ seudomonas aeruginosa in murine thigh and lung infection models. Antimicrob Agents Chemother 54: 1117-1124.

39. (2013) Tobramycin for injection. Prescribing Information.

40. (2013) Amikacin for injection. Prescribing Information.

41. Drusano GL, Louie A (2011) Optimization of aminoglycoside therapy Antimicrob Agents Chemother 55: 2528-2531. 
Citation: Connors KP, Kuti JL, Nicolau DP (2013) Optimizing Antibiotic Pharmacodynamics for Clinical Practice. Pharmaceut Anal Acta 4: 214. doi:10.4172/2153-2435.1000214

42. Nicolau DP, Freeman CD, Belliveau PP, Nightingale CH, Ross JW, et al. (1995) Experience with a once-daily aminoglycoside program administered to 2,184 adult patients. Antimicrob Agents Chemother 39: 650-655.

43. Rea RS, Capitano B, Bies R, Bigos KL, Smith R, et al. (2008) Suboptimal aminoglycoside dosing in critically ill patients. Ther Drug Monit 30: 674-681.

44. Finell DL, Davis GA, Cropp CD, Ensom MH (1998) Validation of the Hartford nomogram in trauma surgery patients. Ann Pharmacother 32: 417-421.

45. Xuan D, Nicolau DP, Nightingale CH (2004) Population pharmacokinetics of gentamicin in hospitalized patients receiving once-daily dosing. Int J Antimicrob Agents 23: 291-295.

46. Buijk SE, Mouton JM, Gyssens IC, Verbrugh HA, Bruining HA (2002) Experience with a once-daily dosing program of aminoglycosides in critically ill patients. Intensive Care Med 28: 936-942.

47. Wolfe T, Dasta J (1998) Comment: once-daily aminoglycoside dosing in critically ill patients. Ann Pharmacother 32: 1109-1110.

48. Kashuba AD, Bertino JS Jr, Nafziger AN (1998) Dosing of aminoglycosides to rapidly attain pharmacodynamic goals and hasten therapeutic response by using individualized pharmacokinetic monitoring of patients with pneumonia caused by gram-negative organisms. Antimicrob Agents Chemother 42: 18421844.

49. Wallace AW, Jones M, Bertino JS Jr (2002) Evaluation of four once-daily aminoglycoside dosing nomograms. Pharmacotherapy 22: 1077-1083.

50. Chuck SK, Raber SR, Rodvold KA, Areff D (2000) National survey of extendedinterval aminoglycoside dosing. Clin Infect Dis 30: 433-439.

51. DeRyke CA, Kuti JL, Nicolau DP (2007) Re-evaluation of current susceptibility breakpoints for Gram-negative rods based on pharmacodynamics assessment Diagn Microbiol Infect Dis 58: 337-344.

52. Nicasio AM, Ariano RE, Zelenitsky SA, Kim A, Crandon JL, et al. (2009) Population pharmacokinetics of high-dose, prolonged infusion cefepime in adult critically ill patients with ventilator-associated pneumonia. Antimicrob Agents Chemother 53:1476-1481.

53. Crandon JL, Ariano RE, Zelenitsky SA, Nicasio AM, Kuti JL, et al. (2011) Optimization of meropenem dosage in the critically ill population based on renal function. Intensive Care Med 37: 632-638.

54. Grant EM, Kuti JL, Nicolau DP, Nightingale C, Quintiliani R (2002) Clinical efficacy and pharmacoeconomics of a continuous-infusion piperacillintazobactam program in a large community teaching hospital. Pharmacotherapy 22: 471-483.

55. Kim A, Sutherland CA, Kuti JL, Nicolau DP (2007) Optimal dosing of piperacillintazobactam for the treatment of $P$ seudomonas aeruginosa infections: prolonged or continuous infusion? Pharmacotherapy 27: 1490-1497.

56. Lodise TP Lomaestro B, Drusano GL (2007) Piperacillin-tazobactam for Pseudomonas aeruginosa infection: clinical implications of an extendedinfusion dosing strategy. Clin Infect Dis 44: 357-363.

57. Yost RJ, Cappelletty DM (2011) The Retrospective Cohort of ExtendedInfusion Piperacillin-Tazobactam (RECEIPT) study: a multicenter study. Pharmacotherapy 31: 767-775.

58. Falagas ME, Tansarli G, Ikawa K, Vardakas KZ (2013) Clinical outcomes with extended or continuous infusion versus short-term intravenous infusion of carbapenems and piperacillin/tazobactam: a systematic review and metaanalysis. Clin Infect Dis 56: 272-282.

59. Dulhunty JM, Roberts JA, Davis JS, Webb SAR, Bellomo R, et al. (2013) Continuous infusion of beta-lactam antibiotics in severe sepsis: a multicenter double-blind, randomized controlled trial. Clin Infect Dis 56: 236-244.

60. Lorente L, Lorenzo L, Martín MM, Jiménez A, Mora ML (2006) Meropenem by continuous versus intermittent infusion in ventilator-associated pneumonia due to gram-negative bacilli. Ann Pharmacother 40: 219-223.

61. Lorente L, Jimenez A, Martin MM, Iribarren JL, Jimenez JJ, et al. (2009) Clinical cure of ventilator-associated pneumonia treated with piperacillin/tazobactam administered by continuous or intermittent infusion. Int J Antimicrob Agents 33: 464-468.

62. Roberts JA, Boots R, Rickard CM, Thomas P, Quinn J, et al. (2007) Is continuous infusion ceftriaxone better than once-a-day dosing in intensive care? A randomized controlled pilot study. J Antimicrob Chemother 59: 285291.

63. Chastre J, Wunderink R, Prokocimer P, Lee M, Kaniga K, et al. (2008) Efficacy and safety of intravenous infusion of doripenem versus imipenem in ventilatorassociated pneumonia: a multicenter, randomized study. Crit Care Med 36: 1089-1096.

64. Kollef MH, Chastre J, Clavel M, Restrepo MI, Michiels B, et al. (2012) A randomized trial of 7-day doripenem versus 10-day imipenem-cilastatin for ventilator-associated pneumonia. Crit Care 16: R218.

65. Nicasio AM, Eagye KJ, Nicolau DP, Shore E, Palter M, et al. (2010) Pharmacodynamic-based clinical pathway for empiric antibiotic choice in patients with ventilator-associated pneumonia. J Crit Care 25: 69-77.

66. Sun HK, Kuti JL, Nicolau DP (2005) Pharmacodynamics of antimicrobials for the empirical treatment of nosocomial pneumonia: a report from the OPTAMA program. Crit Care Med 33: 2222-2227.

67. Deryke CA, Kuti JL, Nicolau DP (2007) Pharmacodynamic target attainment of six beta-lactams and two fluoroquinolones against $P$ seudomonas aeruginosa, Acinetobacter baumannii, Escherichia coli, and Klebsiella species collected from United States intensive care units in 2004. Pharmacotherapy 27: 333-342.

68. Noreddin AM, Marras TK, Sanders K, Chan CK, Hoban DJ, et al. (2004) Pharmacodynamic target attainment analysis against Streptococcus pneumoniae using levofloxacin $500 \mathrm{mg}, 750 \mathrm{mg}$ and $1000 \mathrm{mg}$ once daily in plasma $(P)$ and epithelial lining fluid (ELF) of hospitalized patients with community acquired pneumonia. Int J Antimicrob Agents 24: 479-484.

69. Noreddin AM, Reese AA, Ostroski M, Hoban DJ, Zhanel GG (2007) Comparative pharmacodynamics of garenoxacin, gemifloxacin, and moxifloxacin in community-acquired pneumonia caused by Streptococcus pneumoniae: a Monte Carlo simulation analysis. Clin Ther 29: 2685-2689.

70. Garonzik SM, Li J, Thamlikitkul V, Paterson DL, Shoham S, et al. (2011) Population pharmacokinetics of colistin methane sulfonate and formed colistin in critically ill patients from a multicenter study provide dosing suggestions for various categories of patients. Antimicrob Agents Chemother 55: 3284-3294.

71. Moellering RC Jr (1994) Monitoring serum vancomycin levels: climbing the mountain because it is there? Clin Infect Dis 18: $544-546$.

72. Rybak M, Lomaestro B, Rotschafer JC, Moellering R Jr, Craig W, et al. (2009) Therapeutic monitoring of vancomycin in adult patients: a consensus review of the American Society of Health-System Pharmacists, the Infectious Diseases Society of America, and the Society of Infectious Diseases Pharmacists. Am J Health Syst Pharm 66: 82-98.

73. Patel N, Pai MP, Rodvold KA, Lomaestro B, Drusano GL, et al. (2011) Vancomycin: we can't get there from here. Clin Infect Dis 52: 969-974.

74. van Hal SJ, Lodise TP, Paterson DL (2012) The clinical significance of vancomycin minimum inhibitory concentration in Staphylococcus aureus infections: a systematic review and meta-analysis. Clin Infect Dis 54: 755-771.

75. Lodise TP, Lomaestro B, Graves J, Drusano GL (2008) Larger vancomycin doses (at least four grams per day) are associated with an increased incidence of nephrotoxicity. Antimicrob Agents Chemother 52: 1330-1336.

76. Vandecasteele SJ, De Vriese AS, Tacconelli E (2012) The pharmacokinetics and pharmacodynamics of vancomycin in clinical practice: evidence and uncertainties. J Antimicrob Chemother 68: 743-748. 